\title{
Applying the COM-B Model to Assess the Usability of Smartphone-Connected Listening Devices in Adults with Hearing Loss
}

DOI: $10.3766 /$ jaaa.18061

\author{
David W. Maidment* ${ }^{*}$ \\ Yasmin H. K. Ali*† \\ Melanie A. Ferguson*††
}

\begin{abstract}
Background: Unlike conventional hearing aids, smartphone-connected listening devices may require limited or no input from a trained audiologist in terms of device programming and adjustment. However, there is a lack of peer-reviewed evidence assessing the real-world perspectives of people living with hearing loss toward such technological innovations.
\end{abstract}

Purpose: This study assessed the everyday experiences of adults living with hearing loss toward a range of smartphone-connected listening devices using the Capability, Opportunity, Motivation, Behaviour (COM-B) model as a theoretical framework.

Research Design: A qualitative study where participants trialed one of the following smartphoneconnected listening devices for two weeks in their everyday lives: made-for-smartphone hearing aid, personal sound amplification product, and smartphone "hearing aid" app with wired earphones or wireless hearable. Individual semistructured interviews were conducted.

Study Sample: Twenty adults ( 13 male and 7 female; mean age $=62.25$ years, SD $=11.59$ ) with mildto-moderate hearing loss (mean better ear pure-tone average $=30.49 \mathrm{~dB} \mathrm{HL}, \mathrm{SD}=17.51$ ) were recruited using a convenience sampling strategy. All participants owned conventional hearing aids.

Data Analysis: The data were analyzed using an established deductive thematic analysis procedure within the context of the COM-B model. The model stipulates that for individuals to engage in a particular behavior (B), they must have sufficient capability $(\mathrm{C})$, opportunity $(\mathrm{O})$, and motivation $(\mathrm{M})$.

Results: Capability: One of the key advantages facilitating use and adherence of smartphone-connected listening devices was the ability for participants to make fine-tune adjustments in any listening situation. Opportunity: Participants commented that these devices could address issues surrounding stigma as smartphones are ubiquitous in everyday life. Motivation: Participants consistently reported that the ability to make adjustments via a smartphone provided them with a greater sense of autonomy and empowerment. As a result, they felt more in control of their hearing loss.

Conclusions: This study lays the foundation for further high-quality research to explore whether smartphoneconnected technologies have the potential to yield optimum benefits for people living with hearing loss.

Key Words: COM-B model, hearing aids, hearing loss, smartphone-connected listening devices, Theoretical Domains Framework

Abbreviations: App = Application; $\mathrm{BRC}=$ Biomedical Research Centre; COM-B = Capability, Opportunity, Motivation, Behaviour; HHIE = Hearing Handicap Inventory for the Elderly; NIHR = National Institute for Health Research; PSAP = personal sound amplification product; TDF = Theoretical Domains Framework

*National Institute for Health Research (NIHR) Nottingham Biomedical Research Centre, Nottingham, UK; †Hearing Sciences Section, Division of Clinical Neuroscience, School of Medicine, University of Nottingham, Nottingham, UK; $¥$ Nottingham University Hospitals NHS Trust, Queens Medical Centre, Nottingham, UK

Corresponding author: David W. Maidment, School of Sport, Exercise and Health Sciences, Loughborough University, Loughborough, LE11 3TU, UK; Email: d.w.maidment@Iboro.ac.uk

This paper presents independent research funded by the NIHR Nottingham Biomedical Research Centre and carried out at the NIHR Nottingham Clinical Research Facilities. The views expressed are those of the authors and not necessarily those of the NHS, the NIHR, or the Department of Health and Social Care. 


\section{INTRODUCTION}

$\mathrm{C}$ onventional hearing aids are effective, improving hearing-specific health-related quality of life, listening abilities, and general health-related quality of life in adults living with mild-to-moderate hearing loss (Ferguson et al, 2017). Consequently, the evidence is consistent with the assertion that hearing aids should be routinely offered to individuals who experience hearing and communication difficulties. Nevertheless, most adults who would benefit from using hearing aids fail to access them (Davis et al, 2007; Gopinath et al, 2011; Chien and Lin, 2012). For individuals who do obtain hearing aids, estimates of nonuse vary from $3 \%$ to $24 \%$ (Ferguson et al, 2017). Nonuse and suboptimal use of hearing aids is problematic, not least because untreated hearing loss can result in continued communication difficulties, social isolation, and reduced quality of life for both the individual and their frequent communication partners (Kamil and Lin, 2015; Barker et al, 2017; Vas et al, 2017).

Limited access to and affordability of hearing health care have been cited as key reasons why adults do not seek help for their hearing loss (The National Academies of Sciences Engineering \& Medicine, 2016). In addition to high costs associated with hearing health care to the individual, another identified barrier to accessibility includes a lack of awareness or uncertainty in terms of how to access hearing health care, which may be compounded by clinic-based service delivery models that do not meet the needs and/or preferences of everyone living with hearing loss (Lin et al, 2016). On this basis, there is a clear need to identify alternative service delivery models that could reduce these barriers.

Globally, the use of smartphone technologies in older adults is increasing year-on-year. In the United Kingdom, smartphone ownership has risen exponentially in 55 + year olds, from 19\% in 2012 to $71 \%$ in 2017 (Deloitte, 2017). A similar rise in smartphone ownership of 50-64 year olds has also be found in the United States, rising from 34\% in 2012 to $73 \%$ in 2018 (Pew Research Center, 2018). Furthermore, over the last decade, there has been a proliferation of alternative devices to conventional hearing aids, which can connect wirelessly via Bluetooth to smartphones. Smartphone-connected listening devices include made-for-smartphone hearing aids, personal sound amplification products (PSAPs), and smartphone "hearing aid" applications (or apps). Unlike conventional hearing aids, smartphone-connected listening devices may require limited or no input from a trained audiologist in terms of device programming and adjustment. These devices allow the user to adjust and personalize their hearing programs (e.g., gain and frequency response) via an accompanying smartphone app. As such, smartphone-connected listening devices could address barriers surrounding accessibility and affordability that prevent people from successfully managing their hearing loss. PSAPs and smartphone "hearing aid" apps, for instance, can be purchased at a relatively low cost directly by the user. In comparison with conventional hearing aids, PSAPs also provide comparable electroacoustic characteristics (Callaway and Punch, 2008; Reed et al, 2017b) and speech-in-noise performance (Reed et al, 2017a). Similar findings have also been shown for smartphone "hearing aid" apps (Amlani et al, 2013). However, a recent systematic review and meta-analysis revealed that there is no high-quality evidence assessing the clinical effectiveness of smartphone-connected listening devices (Maidment et al, 2018).

An insight into user experiences of made-for-smartphone hearing aids has been provided in a qualitative study by $\mathrm{Ng}$ et al (2017). They found that, although adults living with hearing loss experienced problems related to Bluetooth connectivity, participants reported that made-for-smartphone hearing aids increased opportunities for social participation in their everyday lives. However, in comparison with PSAPs and smartphone "hearing aid" apps, made-for-smartphone hearing aids are initially programmed to an individual's prescriptive target by a trained audiologist. Further investigation is warranted to determine whether a similar pattern of findings would be found for other smartphone-connected devices that can be purchased and programmed directly by the user.

On this basis, the present study aimed to qualitatively examine the everyday experiences of people living with hearing loss toward a range of smartphone-connected listening devices, using the Capability, Opportunity, Motivation, Behaviour (COM-B) model (Michie et al, 2011) as an underpinning theoretical framework (see Figure 1). The application of theories and models from health psychology has become increasingly prevalent in a hearing health context (e.g., Laplante-Lévesque et al, 2013; Saunders et al, 2013; Meister et al, 2014; Barker et al, 2016; Ferguson et al, 2016; Heffernan et al, 2016). Nevertheless, Coulson et al (2016) argue that popular models used in the field of audiology, such as the Health Belief Model (Rosenstock, 1966), Theory of Planned Behaviour (Fishbein and Ajzen, 1977), and Transtheoretical Model

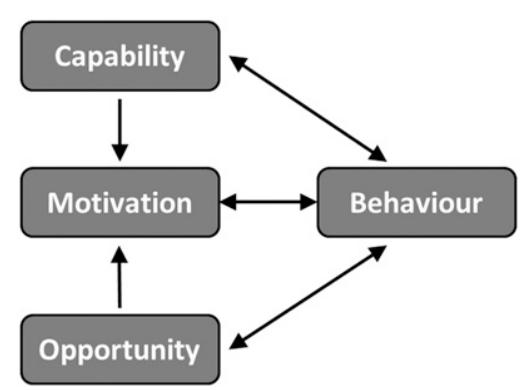

Figure 1. The COM-B model of behavior change. 
(Prochaska and DiClemente, 1983), have been widely criticized because they are inadequate at reliably explaining variations in complex human behavior. By comparison, the COM-B model is a contemporary, supratheory of behavior change, which can enable researchers to understand and describe patient behavior to improve clinical practices within audiology (Coulson et al, 2016). The model has been successfully applied to develop interventions in adult aural rehabilitation (Barker et al, 2016; Ferguson et al, 2019).

The COM-B model stipulates that for individuals to engage in a particular behavior (B), they must have sufficient capability $(\mathrm{C})$, opportunity $(\mathrm{O})$, and motivation $(\mathrm{M})$ (Michie et al, 2011). Capability is defined as an individual's ability to physically and psychologically engage in or perform the behavior. Opportunity refers to external factors that make the behavior possible or prompt it. Motivation is a broad construct that includes basic drives and automatic processes, as well as reflective processes. The COM-B model can also be incorporated into a larger system, the Behaviour Change Wheel (Michie et al, 2011; Cane et al, 2012; Michie et al, 2014), which includes the Theoretical Domains Framework (TDF). The TDF consists of a number of different constructs, such as knowledge, skills, and social influences (see Supplemental Table $\mathrm{S} 1$, available with the online version of this article, for full list), that are necessary to bring about behavior change. In combination, the TDF and the COM-B model can be used to explain why an individual does or does not engage in a particular health-related behavior. In addition, they can facilitate the identification of specific components (e.g., knowledge, skills, etc.) that need to be addressed to bring about a change in that behavior. Thus, in combination, the TDF and the COM-B model can be used to describe and understand the essential components (or active ingredients) of complex healthcare interventions (e.g., hearing aids and smartphoneconnected listening devices).

In this study, the behavior of interest was the use of a smartphone-connected listening device to manage hearing loss in the real-world. The main aim of this study was to identify the factors from the TDF that influence the use of smartphone-connected listening devices in general. The secondary aim was to determine how these factors differed between four different smartphoneconnected listening devices (made-for-smartphone hearing aid, PSAP, and smartphone "hearing aid" app with wired earphones or wireless hearable).

\section{METHODS}

\section{Participants}

A convenience sampling strategy was used (Patton, 1990). Twenty adults were recruited from the National Institute for Health Research (NIHR) Nottingham Bio- medical Research Centre (BRC) participant database, which contains details of people living with hearing loss and/or hearing aid users who have consented to be approached for research. Demographic information of the sample is provided in Table 1. Overall, participants presented with mild-to-moderate hearing loss. All participants owned conventional hearing aids and were experienced users; mean duration of hearing aid ownership was 6.9 years $(\mathrm{SD}=8.9$ years $)$.

\section{Procedure}

All participants attended a one-hour session at the NIHR Nottingham BRC, where written informed consent was obtained. Pure-tone air conduction thresholds were measured at octave frequencies $(0.25-8$ $\mathrm{kHz}$ ) for each ear following the procedure recommended by the British Society of Audiology (2011). In addition, the Hearing Handicap Inventory for the Elderly (HHIE, Ventry and Weinstein, 1982) was completed. HHIE questions were asked as though participants were not wearing their hearing aids. An equal number of participants $(n=5)$ were then randomly assigned to one of the four smartphoneconnected listening device groups. Common to all devices was that they could be controlled using a smartphone app (see also, Figure 2). Hearing aid fitting procedures recommended by the device manufacturer were followed for each device as follows:

\section{Made-for-Smartphone Hearing Aid}

Behind-the-ear Starkey Halo i110 hearing aids (Figure 2A) were individually programmed using InspireX 2016.2 software as directed by the manufacturer (http:// www.starkeyhearingtechnologies.com/inspirehelp/aah) according to the NAL-NL2 algorithm. Hearing aids were fitted with either custom earmolds or open-fit slim tubes, depending on the participant's hearing thresholds.

\section{PSAP}

In-the-ear Starkey Personal Hearing Amplifiers (AMP) (Figure 2B) were programmed using the accompanying smartphone app. In accordance with the manufacturer's guidance, the participants wore foam-padded over-ear headphones during fitting. The AMP was initially adjusted using dual-tone multifrequency signals generated by the smartphone app. One of three preset starting points, corresponding to mild, mild-to-moderate, or moderate sloping hearing loss, was first selected by the first author based on the participant's audiogram. Participants then listened to the media available within the app (adult female speech, adult male speech, restaurant conversation, and music). Participants made adjustments 
Table 1. Demographic Information of Included Participants

\begin{tabular}{lc}
\hline Gender & $\mathrm{N}$ \\
Male & 13 \\
Female & 7 \\
Age & Years \\
Mean & 62.25 \\
SD & 11.59 \\
Range & 21 to 81 \\
Better ear pure-tone average $(0.25-4 \mathrm{kHz})$ & $\mathrm{dB} \mathrm{HL}$ \\
Mean & 30.49 \\
SD & 17.51 \\
Range & 3.4 to 65 \\
Estimated hearing loss duration & Years \\
Mean & 16.41 \\
SD & 13.96 \\
Range & $\leq 1$ to 45 \\
Employment status & $\mathrm{N}$ \\
Retired & 15 \\
Employed & 4 \\
Student & 1 \\
HHIE (unaided) & Total score \\
Mean & 54.20 \\
SD & 11.02 \\
Range & 35 to 70 \\
\hline
\end{tabular}

to low-frequency gain, high-frequency gain, overall gain, and/or output as needed.

\section{Smartphone "Hearing Aid" app with Wireless Hearable}

The Petralex smartphone app (http://petralex.pro/) required participants to first complete an in-app audiometric test. The test includes pure-tone octave frequencies $(0.125-8 \mathrm{kHz})$, which are presented to each ear individually, with the user signaling when the tone becomes audible. On completion of the test, the user can save the test settings as a listening "profile." Microphone selectivity, gain, and frequency response can then be adjusted.

Participants were provided a wireless hearable to use with the Petralex app (Figure 2C). The wireless hearable (The Dash by Bragi, https://support.bragi. com/hc/en-us/categories/200470531-The-Dash) was paired with the user's smartphone via Bluetooth and included additional functionalities, such as health monitoring (e.g., heart rate) and activity tracking (e.g., step count).

\section{Smartphone "Hearing Aid" app with Wired Earphones}

Identical to the wireless hearable described previously, with the exception that participants were instructed to use the Petralex smartphone "hearing aid" app with wired earphones provided with their smartphone (Figure 2D).
All participants trialed the assigned smartphoneconnected listening device in their everyday lives for a period of two weeks. Participants then attended a second session at the NIHR Nottingham BRC, where they were interviewed by the first author. The interview schedule was flexible because of the semistructured design of the interviews, although the core content remained the same across each (see Supplemental Table S2, available with the online version of this article). The interviews were conducted face-to-face in a quiet room and lasted approximately one hour. Each interview was audio-recorded and subsequently transcribed verbatim. Participants were paid a nominal inconvenience fee and travel expenses to attend each study session. The research was approved by the Faculty of Medicine and Health Sciences Research Ethics Committee, University of Nottingham, UK.

\section{Data Analysis}

QSR International's NVivo 10 Software was used to organize and support the analysis of the data. Anonymized identification codes were assigned to each participant (e.g., P1, P2, etc.). The semistructured interview data were analyzed using Braun and Clarke's (2006) thematic analysis procedure. The analysis was deductive (or theoretical), as themes were derived from the components of the TDF, which each link to a specific determinant of behavior (capability, opportunity, and motivation) (Atkins et al, 2017). Furthermore, this approach is suited to answering a specific research question (Braun and Clarke, 2006). To enhance the rigor of the analysis and to ensure that the interpretation of the data was not limited to the perspective of the first author, a second trained researcher (Y.H.K.A.) independently coded all transcripts (Yardley, 2008). Any discrepancies were discussed and an agreement was made regarding which codes should be applied. Themes were derived across all smartphone-connected listening devices. Themes were refined and defined through reanalysis of the data and discussions among the coauthors (Braun and Clarke, 2006). The extent to which these themes enabled the target behavior (i.e., use of a device to manage hearing loss) for each individual listening device was also identified.

\section{RESULTS}

\section{Themes across all Devices}

A summary of the key themes, classified according to the TDF, that were most salient across all smartphoneconnected listening devices is provided in Table 2. Each domain was mapped to the relevant source of behavior (capability, opportunity, and motivation) on the COM-B model. 


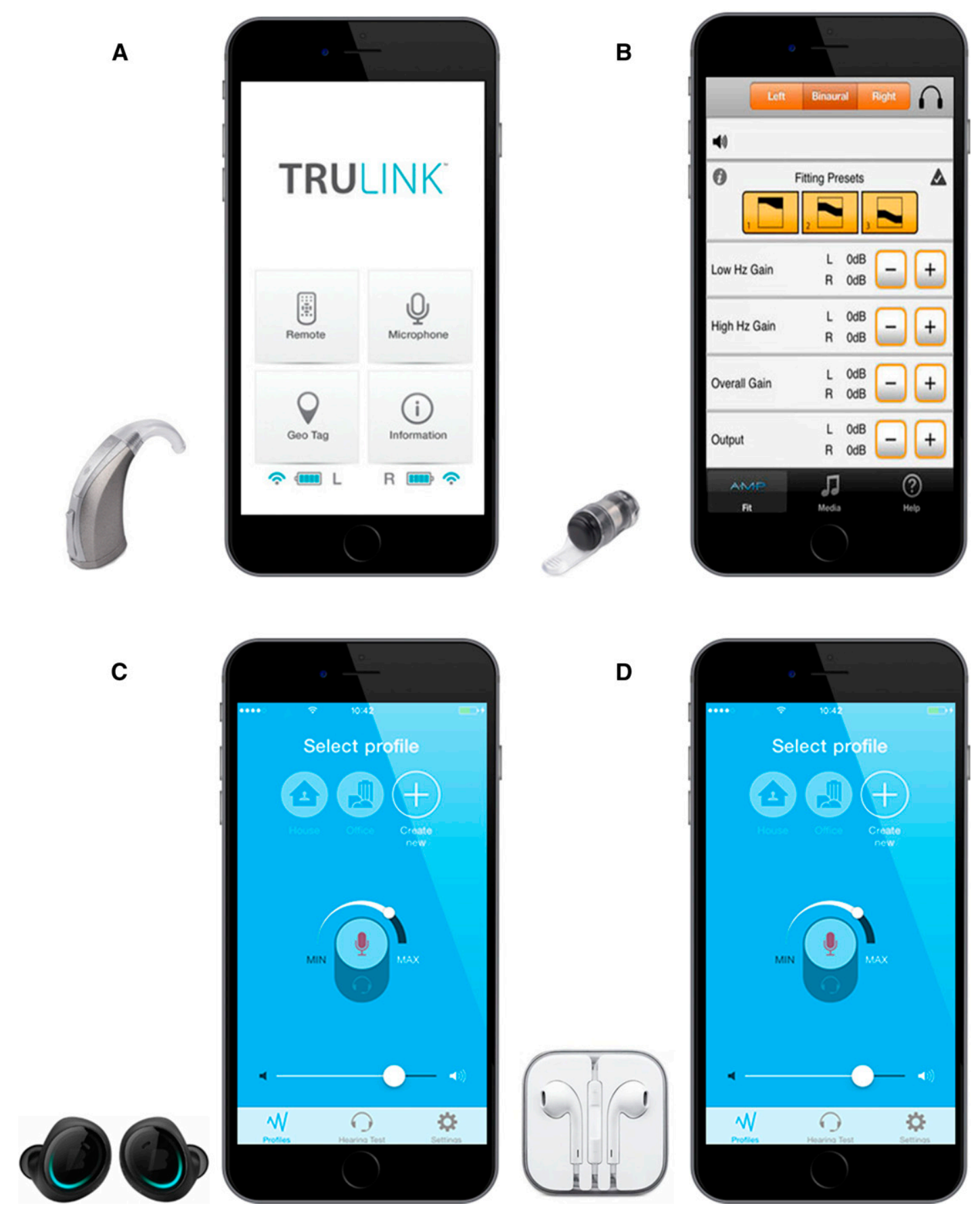

Figure 2. Images of the smartphone-connected listening devices trialed in the study. (A) Smartphone-connected hearing aids: behindthe-ear Starkey Halo i110 hearing aids, adjusted via the TruLink app. (B) Personal sound application product: in-the-ear Starkey AMP Personal Amplifiers, programmed and adjusted using dual-tone multifrequency signals via the accompanying AMP app. (C) Smartphone "hearing aid" app with wireless hearable: the Petralex smartphone app used with Bragi Dash wireless earphones that pair with the user's smartphone via Bluetooth. (D) Smartphone "hearing aid" app with wired earphones: the Petralex smartphone app used with standard wired earphones provided with the user's smartphone. (This figure appears in color in the online version of this article.)

\section{Capability}

\section{Skills}

Digital Literacy: Most participants reported that they required necessary skills in terms of computer and dig- ital literacy to use a smartphone-connected listening device. P1 (made-for-smartphone hearing aid) said: "I use computers a lot; I always have done with my job, so it's very easy for me." Conversely, participants reported that a lack of skills in this area would have a detrimental impact on use. P3 (made-for-smartphone hearing 
aid) commented: "I think that anyone who is not technically astute would have difficulty in connecting-up and understanding the settings and how to adjust them." Thus, the importance of existing digital literacy skills influenced the perceived usability of the listening device trialed.

\section{Knowledge}

Awareness: All participants reported that they had a general lack of awareness of smartphone-connected listening devices before taking part in the study. Some participants reported that they would have liked more information about smartphone-connected devices. P9 (made-for-smartphone hearing aid) said: "Nobody's ever said to me, 'Do you know that there are other devices available?' That's the thing, unless you actually go searching for it, which implies that you need to know that there is something out there in the first place." Other participants reported that they assumed that alternatives to conventional hearing aids were not available for hearing loss. P10 (smartphone "hearing aid" app with wired earphones) explained: "It never crossed my mind that you could use apps to help with hearing loss. I know you can get health apps and stuff like that, but I've always seen it [hearing loss] as a niche thing." Taken together then, participants in the study reported that they might have considered using a smartphoneconnected listening device if they were made more aware that they are available as a management option for hearing loss.

\section{Memory, Attention, and Decision Processes}

Device Simplicity (Plug-and-Play): Adherence to use the device was influenced by whether the smartphoneconnected listening device was viewed as simple and intuitive to use. If the device was perceived as unnecessarily complex or overly sophisticated, participants stated that they were less inclined to persevere using the device. P5 (smartphone "hearing aid" app with wireless hearable) said: "Are they more sophisticated than they need to be? I would say that if you were going to use them just for hearing loss then I suspect they have many more menu features than you really require." In addition, P7 (smartphone "hearing aid" app with wireless hearable) commented: "If it doesn't plugand-play, then you've really got to sit down for half a day and start going in depth to the instruction manual and people are less tolerant to try and sort things out themselves." Moreover, all participants expected the smartphone-connected listening device to be straightforward to set up, and with minimal instruction. P6 (made-for-smartphone hearing aid) said: "It has to be a good piece of hardware, you switch it on and it works."

Satisfaction with Existing Hearing Aids: All participants made comparisons between the smartphone-connected listening device trialed and their conventional hearing aids. Several participants who were already satisfied with their hearing aids reported that they were less likely to use the device. P8 (PSAP) said: "I found it difficult, well, not difficult, just more involved putting them in to your ear. If it's not broke don't fix it. So my current hearing aids work pretty well for me and so I stuck with them." The smartphone-connected listening device was viewed more favorably if participants were less satisfied with their hearing aids. P12 (smartphone "hearing aid" app with wired earphones) stated: "I was quite surprised how good it was initially. In comparison with my hearing aids, which I don't use because I couldn't get on with them, I found that I was quite surprised by the sound quality, I could pick out individual words which is, you know, obviously a big advantage." Therefore, the degree of

Table 2. A Summary of Themes Generated across All Smartphone-Connected Listening Devices

\begin{tabular}{lll}
\hline COM-B & \multicolumn{1}{c}{ TDF } & \multicolumn{1}{c}{ Subtheme } \\
\hline Capability & Skills & Digital literacy \\
& Knowledge & Awareness \\
& Memory, attention, and decision processes & Simplicity (plug-and-play) \\
& Behavioural regulation & Satisfaction with existing hearing aids \\
& Social influences & Adjustability \\
Opportunity & Environmental context & Perception of others and self (stigma) \\
& & Perceived sound quality \\
& & Comfort \\
& & Portability \\
Motivation & Social role and identity & Affordability \\
& Beliefs about capabilities & Age \\
& Goals & Empowerment \\
\end{tabular}

Note: Themes were mapped onto the TDF, which map onto a specific determinant of behavior (capability, opportunity, and motivation) on the COM-B model. 
satisfaction with existing hearing aids influenced take-up, use, and adherence of the smartphone-connected listening device.

\section{Behavioral Regulation}

Adjustability: In comparison with conventional hearing aids, one of the key advantages facilitating the use of a smartphone-connected listening device was the ability to make fine-tune adjustments to meet individual needs and preferences. P8 (smartphone "hearing aid" app with wireless hearable) commented: "If there's a way of making it more finely tuned to myself then I like that opportunity." All participants felt that the ability to make adjustments was extremely beneficial. P6 (made-for-smartphone hearing aid) explained: "The more you can adjust something and make it better for you, the better!"

\section{Opportunity}

\section{Social Influences}

Perception of Others and Self (Stigma): Participants were cognizant of the stigma attached to hearing loss and hearing aids. Some participants commented that smartphone-connected listening devices could address issues surrounding stigma because smartphones are a part of everyday life, which could encourage people to seek help for their hearing loss sooner. P4 (smartphone "hearing aid" app with wired earphones) said: "The perception of having a hearing aid is not very cool is it really? An app might persuade people who wouldn't look at a hearing aid to actually use something, because they're on their phones all the time, their wearing headphones all the time. It is part of their life isn't it?" However, some older participants expressed concerns that other people would perceive them in a negative manner. This was because they felt that the smartphone-connected listening device trialed might be better suited to a younger demographic. For example, P11 (smartphone "hearing aid" app with wireless hearable) said: "I had issues with my perception of what people thought about me. I felt that I couldn't go outside wearing them because I thought that other people would think I was some sort of old man trying to be down with the kids." Nevertheless, P10 (smartphone "hearing aid" app with wired earphones) reported that the benefits of using a smartphone-connected listening device were more important than the perception of others: "The benefit you would gain from it would outweigh any looks you would get. I'm just going to do what I'm going to do because it helps me out. You have any issues with it, then it's your problem. It doesn't matter about anyone else."

\section{Environmental Context and Resources}

Perceived Sound Quality: Participants were more likely to use the smartphone-connected listening device if they perceived the sound quality to be better than that provided by their conventional hearing aids. P14 (smartphone "hearing aid" app with wireless hearable) remarked: "It was very good. It made listening to the TV and radio much clearer. With normal hearing aids I can hear the loudness of it, but I can't hear the words. When I used the app, I could hear more of the words." Similarly, P3 (made-for-smartphone hearing aid) said: "There is a difference in quality of the sound. The fact that these hearing aids are quantitatively three to four times better than my previous hearing aids makes a massive difference. It has given me one end of the audio spectrum to the other. I'm $100 \%$ convinced they are much better." However, if the sound quality was perceived to be poorer, some participants abandoned using the smartphone-connected listening device altogether, instead opting to use their conventional hearing aids. Overamplification of background sounds was highlighted by numerous participants as having a detrimental impact on perceived sound quality. For example, P8 (smartphone "hearing aid" app with wired earphones) remarked: "They amplified everything, yeah, but they amplified the stuff I didn't want to hear too much. The quality of sound wasn't that great to be fair. It did sound like you're sat in a big hall and the echoing and things like that." Overall, perceived sound quality of the smartphone-connected listening device was one of the most important factors prioritized by the participants.

Comfort: Several participants reported that they frequently used the smartphone-connected listening device because it was comfortable to wear. For example, P9 (made-for-smartphone hearing aid) stated: "They were very comfortable to wear. They went in in the morning and they came out at night." In addition, P11 (smartphone "hearing aid" app with wireless hearable) said: "They were comfortable, I couldn't tell that they were there." By comparison, some participants discontinued using the smartphone-connected listening device if it did not fit within their ears comfortably. P17 (PSAP) commented: "I didn't use them due to the fact that they didn't fit properly. They weren't small enough for my ear canals. That was an obvious problem and the reason for stopping using them." Similarly, P5 (smartphone "hearing aid" app with wireless hearable) said: "I personally found the ear buds a little bit uncomfortable. I almost felt there was a pressure build-up."

Portability: Some participants reported that the smartphone-connected listening device was constrictive or cumbersome when completing everyday tasks. P8 
(smartphone "hearing aid" app with wireless hearable) remarked: "Obviously you've got to carry the tablet round with you if you're going out and about, and that's an issue." Most participants became frustrated if they were required to transport other equipment, namely, the wired earphones connected to the smartphone. This was summarized by P13 (smartphone "hearing aid" app with wired earphones) who said: "It's mainly the transportation of it. The fact that you've got this large device here and wires running to your ears, that's a major problem with it." Consequently, ease of portability was a further consideration affecting the use and adherence of a smartphone-connected listening device.

Affordability: All participants reported that the cost of a smartphone-connected listening device, as well as their current financial situation, would be a determining factor influencing future access and use. P13 (smartphone "hearing aid" app with wired earphones) said: "I'm retired and not earning any money. It depends on how much money you've got as to what you think you can afford. I really can't afford any great amount at the moment." Nevertheless, P1 (made-for-smartphone hearing aid) reported that they would consider purchasing a smartphone-connected listening device if this was the only option available to them: "Some people would not consider it a problem at all because they'd have plenty of cash. I've not got that great a pension, but I suppose if I really had to buy one I would."

\section{Motivation}

\section{Social Role and Identity}

Age: All participants reported that a smartphone-connected listening device might be more accessible to a younger demographic. P10 (smartphone "hearing aid" app with wired earphones) commented: "I think it depends on how old you are personally, not to be ageist. I think, if you're a bit tech-savvy you know how to explore an app and find that quite fun. I think if you are a bit older, maybe you don't, you haven't had a smartphone your whole life, I think it would be quite difficult." Similarly, P7 (smartphone "hearing aid" app with wireless hearable) said: "The younger generation tend to use tablets and texting more. I think my generation would find it [the smartphone-connected listening device] just a bit irritating." On this basis, participants felt that smartphone-connected listening devices were better suited to younger individuals because they have greater experience interacting with and using smartphone technologies.

\section{Beliefs about Capabilities}

Empowerment: All participants reported that the ability to make adjustments to the smartphone-connected lis- tening device provided them with a greater sense of autonomy. P4 (smartphone "hearing aid" app with wired earphones) said: "It's the independence, it's that it gives you the control, doesn't it? So if it works for you that's really important because you can control it and adjust it yourself. I like to be able to manage things for myself, where I can." In addition, P9 (made-for-smartphone hearing aid) commented: "It's great. It gives you control. I can actually control my work more because I can actually hear what people are saying to me. So, it gives me that bit of control, and it's not other people running my life, it's me. It's not quality of life, it's just having a life." The ability to make adjustments empowered participants to feel more in control of their hearing loss, resulting in less frustration, greater participation, and more device use.

\section{Goals}

Improved Hearing: All participants were highly motivated to use the smartphone-connected listening devices to improve their hearing and listening abilities. Smartphone-connected listening devices that achieved this were regarded positively. For example, P9 (madefor-smartphone hearing aid) said: "Suddenly I heard this noise, and I thought, 'What the hell is that?' and I realised my husband was using the hairdryer upstairs. I'd never heard the hairdryer from downstairs. So, it just, I can't even describe how much better it made things." Similarly, P10 (smartphone "hearing aid" app with wired earphones) remarked: "I think, for me, the clarity it gave me. It was just so easy to kind of have a conversation with someone and not be like 'pardon, pardon what was that?' I think for me that, because I haven't had that, and it was quite a monumental." By contrast, if the smartphone-connected listening device did not improve the participant's hearing abilities, they reported that were less likely to continue using it. P4 (smartphone "hearing aid" app with wired earphones) said: "I don't feel that it addressed my sort of hearing problem. I didn't feel that it helped me at all. I felt more encumbered with having to deal with it basically. But again, if it was offering me something in terms of hearing improvement, I am sure I would persist with those practical difficulties."

\section{Differences between Devices}

Of the themes identified, there were distinct differences between smartphone-connected listening devices. The made-for-smartphone hearing aid was viewed most favorably. The smartphone "hearing aid" app with wired earphones and PSAP were also viewed positively, but to a lesser extent. The device that had the least positive reviews was the smartphone "hearing aid" app with wireless hearable. 


\section{Made-for-Smartphone Hearing Aids}

All participants reported that they had the necessary skills to use the made-for-smartphone hearing aid, as well as the accompanying app, because they were both straightforward to use: "These were very quick and easy to use" (P3). In addition, participants commented that they could easily make adjustments using the app to improve their ability to hear, particularly in noisy environments. As a consequence, participants consistently stated that this gave them a greater sense of control, empowering them to manage their hearing loss in their everyday lives. This resulted in less frustration and greater selfreported device use: "I think the device is absolutely fantastic. They've improved my quality of life no end. It's that stopping you from wanting to burst into tears of sheer frustration. I feel much more confident now surprisingly enough" (P9). All participants also felt that the made-forsmartphone hearing aids were superior in terms of sound quality in comparison with their conventional hearing aids that they could not adjust themselves. Nevertheless, participants were mindful that made-for-smartphone hearings aids are not currently available free-of-charge from the publically funded UK's National Health Service (NHS). Therefore, they were uncertain whether they would be able to financially afford these devices: "I feel that I can't be spending this amount of money" (P9).

\section{Smartphone "Hearing Aid" app with Wired Earphones}

Most participants commented that this device was simple and intuitive to use and required very little instruction to set up. In relation, participants felt that it was easy to make adjustments and personalize the sound settings to meet their individual needs and/or preferences: "The fact that you could fine-tune it yourself, rather than having to keep going back, is really invaluable" (P4). Moreover, some participants felt that this device was less noticeable and, therefore, reduced the stigma associated with hearing loss. In particular, P10 commented that the use of smartphones and wired earphones are the norm: "It helped a lot and obviously no one looked at me weird because I could just be listening to music.” However, all participants commented that this device was impractical from a portability standpoint, and would impede movement: "It was alright except, as I say, I had to keep picking the thing up and going somewhere with it, which I didn't really think was very good" (P14). Participants also expressed ambivalent views concerning the sound quality. Although some participants reported that the device improved listening in quiet environments, such as watching the television, all participants remarked that the device performed poorly in noisy listening situations, as all sounds were amplified, including the background noise.

\section{PSAP}

All participants who trialed this device felt that the PSAP was difficult to use and adjust using the accompanying smartphone app. All participants expressed concerns regarding the physical fit of the device. For example, $\mathrm{P} 15$ commented that the device moved and often fell-out of their ear: "The only problem was if you are talking a lot or eating then they came out your ear, so you were forever pushing them back in again." In relation, P18 was apprehensive about inserting the device into their ear for fear that it would get stuck, commenting: "I did have difficulty putting them into my ear. I pushed the device too far into the ear canal so it was difficult to retrieve." Despite this, participants commented that they persisted with the device because it was "smaller" and "more discreet" (P19) in comparison with their conventional hearing aids. Similar to the smartphone "hearing aid" app with wired earphones, participants also reported that the device improved listening abilities in quiet environments, but provided little benefit when there was background noise: "In a conversation with two female friends I found it very difficult to hear what they were saying on occasions" (P2).

\section{Smartphone "Hearing Aid" app with Wireless Hearable}

The main issue that all participants reported was the difficulty they experienced pairing the hearable device via Bluetooth to their smartphone. They reported that the device was overly sophisticated or too complex to use: "It depends on how techy savvy you are...my kids generation that have grown up with smartphones, I think they wouldn't bat an eye lid, they would be quite happy and they wouldn't need support, so I think it's a generational thing" (P20). In addition, participants commented that the device was uncomfortable or irritating to wear for a prolonged period of time: "I personally found the ear buds a little bit uncomfortable... I felt, I almost felt there was a pressure build-up" (P5). Furthermore, all users ceased using the device because of the sound delay experienced when using the smartphone "hearing aid" app, because of the wireless transmission of the sound from the smartphone microphone to the hearable. Participants regularly reported that the delay was "irritating" (P8), which reduced confidence in the device: "I was not sensing that I was getting amplification in a way that was tolerable because of that delay, and I just assumed that it wasn't going to do anything for me" (P11).

\section{DISCUSSION}

lthough the last decade has seen a substantial rise in the availability of smartphone-connected 
listening devices, there is a sparsity of peer-reviewed evidence assessing the real-world perspectives of people living with hearing loss toward such technological innovations. To date, only one published study has aimed to understand how smartphone connectivity can influence patient experiences and clinician practices, focusing solely on made-for-smartphone hearing aids $(\mathrm{Ng}$ et al, 2017). The present study contributes further to this evidence base by qualitatively describing the everyday experiences of people living with hearing loss toward a range of smartphone-connected listening devices. In addition, these experiences are explained within the context of the COM-B model and TDF (Cane et al, 2012; Michie et al, 2014), which serve as a theoretical underpinning to gain an insight into the specific determinants of the target behavior (i.e., use of a smartphone-connected listening device to manage hearing loss).

\section{Capability}

With regards to individuals' ability to physically and psychologically use a smartphone-connected listening device to manage their hearing loss, the following five subthemes emerged: (1) the requirement to have necessary digital literacy skills; (2) awareness that these devices are an available management option for hearing loss; (3) the expectation that smartphone connected listening devices should be simple and intuitive to use with minimal instruction (i.e., plug-and-play); (4) the extent to which participants were satisfied (or not) with their existing conventional hearing aids; and (5) the advantages of being able to make adjustments to meet individual listening and communication needs. In terms of each individual listening device, both the made-for-smartphone hearing aids and smartphone "hearing aid" app with wired earphones were straightforward to set up and adjust. In comparison, participants reported that they experienced difficulties when attempting to adjust the PSAP and when pairing the wireless hearable via Bluetooth for use with the smartphone "hearing aid" app.

$\mathrm{Ng}$ et al (2017) similarly found that patients identified themselves as either competent or incompetent technology users. On this basis, the level of technological competence could be used by clinicians as a means to identify suitable candidates for smartphone-connected listening devices. Nevertheless, Ng et al (2017) argue that this assumption may be misguided because patients who identify as less technologically competent may be willing to learn how to successfully use smartphone-connected listening devices. Given that smartphone ownership continues to rise exponentially year-on-year in older adults (Deloitte, 2017), the potential barrier of digital literacy may become less of a concern in the future. Furthermore, a number of studies suggest that digital technologies are accessible to older adults with hearing loss, given that they report higher levels of computer and Internet competency relative to that in the general age-matched population (Henshaw et al, 2012; Thorén et al, 2013).

We also found that participants in this study reported that they lacked awareness that smartphone-connected technologies were an available management option for hearing loss. Likewise, the National Academies of Sciences Engineering \& Medicine (2016) report suggests that one of the key barriers to accessibility of hearing health care in adults is limited knowledge of available treatment options. There is, therefore, a need to better educate people living with hearing loss, as well as the public more generally, on the consequences of hearing loss and how it can be managed. In addition, sufficient training is warranted for general health-care professionals, so that they might screen, refer, and/or educate people living with hearing loss (Lin et al, 2016). Public awareness campaigns are one approach that could address this need, which could also be facilitated through the use of mobile-enabled health (or mHealth) technologies. For example, smartphone-based hearing tests delivered via an app have been deployed in South Africa (https://www.hearxgroup.com/hearscreen/) and, more recently, the United States (https://www.hearscreenusa. com/) to improve hearing loss awareness and subsequent help-seeking.

\section{Opportunity}

The external factors that enabled (or prompted) the use of a smartphone-connected listening device were clustered into the following five subthemes: (1) a reduction in stigma because smartphones are a part of everyday life; (2) the perceived sound quality of the device; (3) the extent to which the device fitted comfortably within the ear; (4) portability of the device when undertaking everyday tasks; and (5) affordability of the device relative to the individual's current financial situation.

Participants reported that the perceived sound quality for made-for-smartphone hearing aids was superior in comparison with participant's existing conventional hearing aids. Although equivalent outcomes have been shown for "basic" and "advanced" hearing aids (Cox et al, 2016; Johnson et al, 2016), this finding likely arose because the made-for-smartphone hearing aids trialed could have been a much higher specification than participant's existing hearing aids. For the other smartphone-connected listening devices, participants expressed ambivalence concerning perceived sound quality. Specifically, some participants reported that although the PSAP and smartphone "hearing aid" app provided sufficient amplification in quiet conditions, they were inadequate in noisy listening situations. By contrast, laboratory-based studies, using behavioral measures of speech intelligibility, have shown similar 
levels of performance between conventional hearing aids and both PSAPs (Reed et al, 2017a) and smartphone "hearing aid" apps (Amlani et al, 2013). Although the potential dissociation between this study and previous findings warrants further investigation, a potential explanation is that people with hearing loss may provide self-reported estimates of speech intelligibility that are significantly lower than performance attained via behavioral measures (Cox et al, 1991). On this basis, caution should be exercised when comparing qualitative reports of perceived sound quality with quantitative measures of speech intelligibility.

We consistently found that smartphone-connected listening devices were viewed as potentially less stigmatizing in comparison with conventional hearing aids. This was primarily attributed to the perception that smartphone technologies are ubiquitous and, as a consequence, less noticeable to others. The stigma associated with hearing loss and the use of hearing aids has been identified as a key barrier to accessing hearing health care (Southall et al, 2010; Wallhagen, 2010; Barker et al, 2017; Vas et al, 2017). For example, people living with hearing loss may be concerned that others will perceive them as socially incompetent and cognitively diminished (Southall et al, 2010). It has been argued that smartphone technologies have the potential to reduce the stigma associated with hearing loss, as well as encourage and empower adults living with hearing loss to seek earlier intervention (Amlani, 2015). Our findings support this notion, with many participants stating that smartphone-connected listening devices would likely be accessed sooner by individuals who might not want to use a hearing aid to manage their hearing loss. However, although stigma can be reduced through the integration of listening devices with smartphone technologies, it has been suggested that this accessibility barrier could be further reduced through the promotion of better societal awareness of hearing loss and hearing aid use (Ng et al, 2017).

\section{Motivation}

Three subthemes were identified under the construct of motivation: (1) the view that smartphone-connected listening devices might be better suited to a younger demographic; (2) the ability to make adjustments empowered users to self-manage their hearing loss; and (3) an expectation that using the device should improve hearing and listening abilities. We consistently found that irrespective of the smartphone-connected listening device trialed, the ability to make adjustments, such as in terms of gain and/or frequency response, gave users a greater sense of autonomy. As such, participants felt more in control of their hearing loss, which made them feel that they participate more fully in social situations. These findings are comparable with those of $\mathrm{Ng}$ et al
(2017), who also found that made-for-smartphone hearing aids increased self-reported opportunities for social participation in everyday life.

A commonly cited reason why people fitted with hearing aids stop using them is because they continue to experience difficulties when listening to and understanding speech in noisy situations (McCormack and Fortnum, 2013). Our findings suggest that the provision of smartphone-connected listening devices may alleviate these difficulties, providing an opportunity for people living with hearing loss to alter their device programming in any situation to address their individual listening and communication needs. It should be noted, however, that the concept of situation-specific personalization of hearing aid programming predates the proliferation of smartphone technologies. For example, the hearing aid programming preferences of the individual have previously been shown to differ depending on the listening environment (Keidser et al, 2005). Furthermore, Dillon et al (2006) introduced the concept of trainable hearing aids that could be optimally adjusted in any situation by the user, and would be capable of learning the user's preferred settings. This study adds to this existent literature, highlighting that it is important for people living with hearing loss to discreetly and conveniently control their own listening devices, such as via a smartphone app. This provides users with a greater sense of control, resulting in less frustration, greater participation, and optimal device use.

\section{Study Limitations and Future Research}

We acknowledge that there are a number of caveats to the design of the present study that could be addressed in future research. For example, we made the pragmatic decision to include only existing hearing aid users, to allow for a comparison between existing conventional hearing aids and smartphone-connected listening device. Participants who experience hearing loss, but have no prior experience of amplification, would potentially be devoid of preexisting bias. Consequently, naïve users could provide alternative advantages and disadvantages to device usage that were not reported in the present study. In addition, for logistical reasons, it was only possible for participants to trial the smartphone-connected listening device for two weeks. Participant's experiences may have been different if they had more time to use and acclimatize to the device. In relation, it is likely that participants had varied listening experiences, which could have influenced the outcomes of the study. Although our aim was to understand the everyday experiences of people living with hearing loss toward smartphone-connected listening devices, information regarding the listening situations encountered by participants could be measured in future studies, such as via hearing diaries, data logging, or ecological 
momentary assessment (for recent application, see Timmer et al, 2018). Logistical reasons also necessitated the use of a convenience sampling strategy, which is potentially less rigorous than other qualitative sampling techniques. Nevertheless, the sample was representative of the target population (i.e., adults living with hearing loss currently using conventional hearing aids).

The present study is the first to evaluate the perspectives of people living with hearing loss toward a range of smartphone-connected listening devices. Recently published guidelines for hearing loss assessment and management by the United Kingdom National Institute for Health and Care Excellence (2018) recommend that randomized controlled trials are needed in the area of assistive listening devices, including smartphone-connected technologies. To that end, the findings from this study could be used to inform further work assessing the clinical- and cost-effectiveness of smartphone-connected listening devices that require limited or no audiological input. In accordance with the UK Medical Research Council guidelines (Campbell et al, 2000; Medical Research Council, 2006), developmental studies involving qualitative methodologies should be used to provide important insights into how health-care interventions operate, which can then be used to refine the robust design of future effectiveness trials. However, further research in this area should also investigate the extent to which adults living with hearing loss require further assistance from a hearing health-care professional, even if audiological input is limited during device programming. Indeed, a randomized, double-blind, placebocontrolled clinical trial has evaluated audiologist programmed and preprogrammed hearing aids (i.e., over-the-counter service delivery model) (Humes et al, 2017). Humes et al (2017) found that, although both types of hearing aid resulted in similar levels of self-reported speech recognition and hearing aid benefit, satisfaction with preprogrammed hearing aids was lower, possibly because of the absence of audiological interaction.

\section{Conclusion}

This study has demonstrated the utility of applying a contemporary model of behavior change, to explain the everyday experiences of adults living with hearing loss toward a range of smartphone-connected listening devices. We have identified the key factors that influence use of a smartphone-connected listening device to manage hearing loss when used by adults in the real world. Overall, we found that people living with hearing loss want to conveniently personalize and adjust their own listening devices via their smartphone to improve their ability to listen and communicate in any situa- tion. Given that smartphone technologies are ubiquitous, they also have the potential to reduce the stigma associated with hearing loss and hearing aids, which could encourage individuals to seek help from an earlier time point, as well as empower them to manage their hearing loss. Although additional evidence is necessary in this area, this study lays the foundation for further research to explore the effectiveness of smartphone-connected listening devices. Such research is needed to determine whether smartphone-connected listening devices have the potential to revolutionize hearing health-care service delivery and provide opportunities for wider accessibility to yield optimum benefits for people living with hearing loss.

Acknowledgments. We thank Dr. Eithne Heffernan and Professor Neil Coulson for their comments on early drafts of the manuscript. We would also like to thank Starkey Hearing Technologies, UK, for providing devices free-of-charge for the purposes of this research.

\section{REFERENCES}

Amlani AM. (2015) Improving patient compliance to hearing healthcare services and treatment through self-efficacy and smartphone applications. Hearing Rev 22:16-20.

Amlani AM, Taylor B, Levy C, Robbins R. (2013) Utility of smartphone-based hearing aid applications as a substitute to traditional hearing aids. Hearing Rev 20:16-18.

Atkins L, Francis J, Islam R, O'Connor D, Patey A, Ivers N, Foy R, Duncan EM, Colquhoun H, Grimshaw JM. (2017) A guide to using the Theoretical Domains Framework of behaviour change to investigate implementation problems. Implement Sci 12:1-18.

Barker A, Leighton P, Ferguson M. (2017) Coping together with hearing loss: a qualitative meta-synthesis of the psychosocial experiences of people with hearing loss and their communication partners. Int $J$ Audiol 56:297-305.

Barker F, Atkins L, de Lusignan S. (2016) Applying the COM-B behaviour model and behaviour change wheel to develop an intervention to improve hearing aid use in adult auditory rehabilitation. Int J Audiol 55:S90-S98.

Braun V, Clarke V. (2006) Using thematic analysis in psychology. Qual Res Psychol 3:77-101.

British Society of Audiology. (2011) Recommended Procedure: Pure-Tone Air-Conduction and Bone-Conduction Threshold Audiometry with and without Masking. Reading, United Kingdom.

Callaway SL, Punch JL. (2008) An electroacoustic analysis of overthe-counter hearing aids. Am J Audiol 17:14-24.

Campbell M, Fitzpatrick R, Haines A, Kinmonth AL, Sandercock P, Spiegelhalter D, Tyrer P. (2000) Framework for design and evaluation of complex interventions to improve health. BMJ 321: 694-696.

Cane J, O'Connor D, Michie S. (2012) Validation of the theoretical domains framework for use in behaviour change and implementation research. Implement Sci 7:37. 
Chien W, Lin FR. (2012) Prevalence of hearing aid use among older adults in the United States. Arch Intern Med 172: 292-293.

Coulson N, Ferguson MA, Henshaw H, Heffernan E. (2016) Applying theories of health behaviour and change to hearing health research: time for a new approach. Int $J$ Audiol 55:S99-S104.

Cox RM, Alexander GC, Rivera IM. (1991) Comparison of objective and subjective measures of speech intelligibility in elderly hearing-impaired listeners. $J$ Speech Lang Hear Res 34: 904-915.

Cox RM, Johnson JA, Xu J. (2016) Impact of hearing aid technology on outcomes in daily life I: the patients' perspective. Ear Hear $37: \mathrm{e} 224-\mathrm{e} 237$

Davis A, Smith P, Ferguson M, Stephens D, Gianopoulos I. (2007) Acceptability, benefit and costs of early screening for hearing disability: a study of potential screening tests and models. Health Technol Assess 11:1-294.

Deloitte. (2017) State of the Smart: Seventh Annual Mobile Consumer Survey. Deloitte Limited Liability Partnership (LLP): London, United Kingdom.

Dillon H, Zakis JA, McDermott H, Keidser G, Dreschler W, Convery E. (2006) The trainable hearing aid: what will it do for clients and clinicians? Hearing $J$ 59:30-36.

Ferguson MA, Coulson NS, Henshaw H, Heffernan E. (2016) Application of health behaviour theory to hearing healthcare research: the state of play and beyond. Int J Audiol 55:S1-S2.

Ferguson MA, Kitterick P, Chong L, Edmondson-Jones M, Barker F, Hoare D. (2017) Hearing aids for mild to moderate hearing loss in adults. Cochrane Database Syst Rev 9:CD012023.

Ferguson MA, Maidment DW, Henshaw H, Gomez R. (2019) Knowledge is power: improving outcomes for patients, partners, and professionals in the digital age. Perspect ASHA Spec Interest Groups 4:140-148.

Fishbein M, Ajzen I. (1977) Belief, Attitude, Intention, and Behavior: An Introduction to Theory and Research. Reading, MA: AddisonWesley.

Gopinath B, Schneider J, Hartley D, Teber E, McMahon CM, Leeder SR, Mitchell P. (2011) Incidence and predictors of hearing aid use and ownership among older adults with hearing loss. Ann Epidemiol 21:497-506.

Heffernan E, Coulson N, Henshaw H, Barry JG, Ferguson MA. (2016) Understanding the psychosocial experiences of adults with mild-moderate hearing loss: a qualitative study applying Leventhal's self-regulatory model. Int J Audiol 55:S3-S12.

Henshaw H, Clark D, Kang S, Ferguson MA. (2012) Computer skills and Internet use in adults aged 50-74 years: influence of hearing difficulties. J Med Internet Res 14:e113.

Humes LE, Rogers SE, Quigley TM, Main AK, Kinney DL, Herring C. (2017) The effects of service-delivery model and purchase price on hearing-aid outcomes in older adults: a randomized doubleblind placebo-controlled clinical trial. Am J Audiol 26:53-79.

Johnson JA, Xu J, Cox RM. (2016) Impact of hearing aid technology on outcomes in daily life II: speech understanding and listening effort. Ear Hear 37:529-540.

Kamil RJ, Lin FR. (2015) The effects of hearing impairment in older adults on communication partners: a systematic review. $J$ Am Acad Audiol 26:155-182.
Keidser G, Brew C, Brewer S, Dillon H, Grant F, Storey L. (2005) The preferred response slopes and two-channel compression ratios in twenty listening conditions by hearing-impaired and normalhearing listeners and their relationship to the acoustic input. Int $J$ Audiol 44:656-670.

Laplante-Lévesque A, Hickson L, Worrall L. (2013) Stages of change in adults with acquired hearing impairment seeking help for the first time: application of the transtheoretical model in audiologic rehabilitation. Ear Hear 34: $447-457$

Lin FR, Hazzard WR, Blazer DG. (2016) Priorities for improving hearing health care for adults: a report from the national academies of sciences, engineering, and medicine. JAMA 316: $819-820$.

Maidment DW, Barker AB, Xia J, Ferguson MA. (2018) A systematic review and meta-analysis assessing the effectiveness of alternative listening devices to conventional hearing aids in adults with hearing loss. Int J Audiol 57:721-729.

McCormack A, Fortnum H. (2013) Why do people fitted with hearing aids not wear them? Int J Audiol 52:360-368.

Medical Research Council. (2006) Developing and Evaluating Complex Interventions: New Guidance. London, United Kingdom.

Meister H, Grugel L, Meis M. (2014) Intention to use hearing aids: a survey based on the theory of planned behavior. Patient Prefer Adherence 8:1265-1275.

Michie S, Atkins L, West R. (2014) The Behaviour Change Wheel:A Guide to Designing Interventions. London, United Kingdom: Silverback.

Michie S, van Stralen M, West R. (2011) The behaviour change wheel: a new method for characterising and designing behaviour change interventions. Implement Sci 6:42.

National Institute for Health and Care Excellence. (2018) Hearing Loss in Adults: Assessment and Management. London, United Kingdom.

Ng SL, Phelan S, Leonard M, Galster JA. (2017) A qualitative case study of smartphone-connected hearing aids: influences on patients, clinicians, and patient-clinician interactions. J Am Acad Audiol 28:506-521.

Patton MQ. (1990) Qualitative Evaluation and Research Methods. Newbury Park, CA: SAGE Publications.

Pew Research Center. (2018) Mobile Fact Sheet. Washington, DC.

Prochaska JO, DiClemente CC. (1983) Stages and processes of self-change of smoking: toward an integrative model of change. J Consult Clin Psychol 51:390-395.

Reed NS, Betz J, Kendig N, Korczak M, Lin FR. (2017a) Personal sound amplification products vs a conventional hearing aid for speech understanding in noise. JAMA 318:89-90.

Reed NS, Betz J, Lin FR, Mamo SK. (2017b) Pilot electroacoustic analyses of a sample of direct-to-consumer amplification products. Otol Neurotol 38:804-808.

Rosenstock IM. (1966) Why people use health services. Milbank Mem Fund Q 44:94-127.

Saunders GH, Frederick MT, Silverman S, Papesh M. (2013) Application of the health belief model: development of the hearing 
beliefs questionnaire (HBQ) and its associations with hearing health behaviors. Int $J$ Audiol 52:558-567.

Southall K, Gagne JP, Jennings MB. (2010) Stigma: a negative and a positive influence on help-seeking for adults with acquired hearing loss. Int J Audiol 49:804-814.

The National Academies of Sciences Engineering \& Medicine. (2016) Hearing Healthcare for Adults: Priorities for Improving Access and Affordability. Washington, DC.

Thorén ES, Öberg M, Wänström G, Andersson G, Lunner T. (2013) Internet access and use in adults with hearing loss. J Med Internet Res 15:e91.

Timmer BH, Hickson L, Launer S. (2018) Do hearing aids address real-world hearing difficulties for adults with mild hearing impair- ment? Results from a pilot study using ecological momentary assessment. Trends Hear 22:1-15.

Vas V, Akeroyd MA, Hall DA. (2017) A data-driven synthesis of research evidence for domains of hearing loss, as reported by adults with hearing loss and their communication partners. Trends Hear 21:1-25.

Ventry IM, Weinstein BE. (1982) The hearing handicap inventory for the elderly: a new tool. Ear Hear 3:128-134.

Wallhagen MI. (2010) The stigma of hearing loss. Gerontologist 50: 66-75.

Yardley L. (2008) Demonstrating validity in qualitative psychology. In: Smith JA, ed. Qualitative Psychology: A Practical Guide to Research Methods. London, United Kingdom: SAGE Publications. 


\section{Supplemental Table S1}

Supplemental 1. Definitions of the Theoretical Domains Framework (TDF: Cane et al., 2012; Michie et al., 2014).

\begin{tabular}{|c|c|c|}
\hline COM-B component & Theoretical Domain & Definition \\
\hline \multirow[t]{4}{*}{ Capability } & Knowledge & An awareness of the existence of something. \\
\hline & Skills & An ability or proficiency acquired through practice. \\
\hline & Memory, attention \& decision processes & $\begin{array}{l}\text { The ability to retain information, focus selectively on aspects of the } \\
\text { environment and choose between two or more alternatives. }\end{array}$ \\
\hline & Behavioural regulation & $\begin{array}{l}\text { Anything aimed at managing or changing objectively observed or } \\
\text { measured actions. }\end{array}$ \\
\hline \multirow[t]{2}{*}{ Opportunity } & Social influences & $\begin{array}{l}\text { Those interpersonal processes that can cause individuals to change their } \\
\text { thoughts, feelings, or behaviours. }\end{array}$ \\
\hline & Environmental context \& resources & $\begin{array}{l}\text { Any circumstance of a person's situation or environment that } \\
\text { discourages or encourages the development of skills and abilities, } \\
\text { independence, social competence, and adaptive behaviour. }\end{array}$ \\
\hline \multirow[t]{8}{*}{ Motivation } & Social/professional role \& identity & $\begin{array}{l}\text { A coherent set of behaviours and displayed personal qualities of an } \\
\text { individual in a social or work setting. }\end{array}$ \\
\hline & Beliefs about capabilities & $\begin{array}{l}\text { Acceptance of the truth, reality, or validity about an ability or facility } \\
\text { that a person can put to a constructive use. }\end{array}$ \\
\hline & Beliefs about consequences & $\begin{array}{l}\text { Acceptance of the truth, reality, or validity about outcomes of a } \\
\text { behaviour in a given situation. }\end{array}$ \\
\hline & Intentions & $\begin{array}{l}\text { A conscious decision to perform a behaviour or a resolve to act in a } \\
\text { certain way. }\end{array}$ \\
\hline & Goals & $\begin{array}{l}\text { Mental representation of outcomes or end states that an individual wants } \\
\text { to achieve. }\end{array}$ \\
\hline & Reinforcement & $\begin{array}{l}\text { Increasing the probability of a response by arranging a dependent } \\
\text { relationship, or contingency, between the response and a given stimulus. }\end{array}$ \\
\hline & Emotion & $\begin{array}{l}\text { A complex reaction pattern, involving experiential, behavioural, and } \\
\text { physiological elements, by which the individual attempts to deal with a } \\
\text { personally significant matter or event }\end{array}$ \\
\hline & Optimism & $\begin{array}{l}\text { The confidence that things will happen for the nest or that desired goals } \\
\text { will be attained. }\end{array}$ \\
\hline
\end{tabular}




\section{Supplemental Table S2}

Supplemental 2. Semi-structured interview schedule.

\section{Main question}

\section{Optional probe questions}

What did you like?

What do you think were the benefits or

Tell me what you thought of the device and how you got on.

advantages?

What didn't you like?

What do you think were the shortcomings or disadvantages?

How often did you use it?

Can you tell me how you used the device? In what situations did you use it?

When did you use it?

Did you feel that you wanted or needed to use it? Why/why not?

Did you get into a habit of using it? Why/why not?

Would you have liked support to use it? If so, from whom and why?

Would you consider buying one? Why/why not?

How much would you consider paying for $i t$ ?

What would make paying for it worthwhile?

If more people used it, would it make you want to use it?

Can you tell me whether you are aware of
anyone else that has used this device? If so, who? 